\title{
Enlightenment of Art Design Education by Ecological Design
}

\author{
Hua-Zheng LIU \\ Communication and animation Institute, Qingdao University of Science and Technology, No.99, \\ Songling Road, Laoshan District, Qingdao, Shandong Province, China \\ E-mail:liuhuazheng1949@163.com
}

Keywords: ecological, design, education

\begin{abstract}
Any form of design coordinated with the ecological process and trying to minimize its destructive impact on the environment can be referred to as an ecological design. Through investigation in the new concept of ecological design as well as the design principle of the entire system etc, this paper points out that design education serves an irreplaceable role for ecological protection, and ecological design can be realized in an all-round way only through the reform of design education, the enhancement of trans-disciplinary intersection and fusion, and the formation of correct ecological values in students etc.

When introduced to the category of design, ecology becomes an important basis and principle for design and a standard for the examination of social development, which also reminds people that all alienation phenomena brought about by civilization must be constantly reevaluated and corrected.

The specialized characteristics of art design have put it at the leading edge of the concept of ecological protection. As designers, we impose tremendous impact on the ecological environment. The constantly increasing awareness of ecological consciousness and ecological values has caused a great stir to the field of design and has become the latest influencing factor of design concept, exerting considerable functions in the works by future designers. For ecological protection, therefore, design education plays an irreplaceable role and the cultivation of designers respecting the natural world and bearing the mind of ecological design can be possible only when the concept of ecological design rungs through the entire process of design education.
\end{abstract}

\section{On Ecological Design}

Any form of design coordinated with the ecological process and trying to minimize its destructive impact on the environment can be referred to as an ecological design which has ecological concerns as its basic restrictive conditions. And to specify, an ecological design studies the sustainable development of material production and the consumption activities of humans based on the relation between "feeling" and "object and environment". An ecological design means respect over species diversity, reduction in waste of resources and maintenance of the quality of plant environment and animal habitats, in order to help improve human habitat environment and the ecological system, and this is a thinking method and an implementation process for humans to handle ecological issues in the field of design

Being a manner for interaction and coordination with the nature, ecological design is not peculiar to any specific profession or subject and has a very wide coverage from an architectural designer's consideration of his design and material selection to a product designer's restraint use of hazardous substances etc. Ecological design provides us a collective framework to help us reevaluate design and people's daily living habits and behaviors. Concerning the effective adaptation to and combination with nature, ecological design makes an overall weighing of changes caused to the nature by design. For each and every design, we need to consider whether or not it helps improve or restore the living world and protect relevant ecological environment structure. 


\section{New Concept of Ecological Design}

Compared with conventional design, ecological design has its new concepts over many design issues, and it's the evolution extension of conventional design, not a mutation or rupture. A design that consumes much energy and lacks cultural implication and sense of beauty will not be accepted by the society, and will eventually be forgotten and overwhelmed; the value of design, therefore, will not be expressed. The concept of ecological design, ultimately, emphasizes the conformability and systematicness of design through a 3D ecological mode of thinking featured by openness, which thus has expanded living space of design, making the design comprehensive in new aspects.

\section{Design Principle of the Entire System-“Annular” Design}

When ecological issues are introduced as restrictive conditions into design, design works must obey ecological principles. Then we have specific ecological indexes for design evaluation, and an ecological design has the concept of "holistic design" instead of "linear design". The "holistic design" emphasizes the recycling of energy resources, low energy consumption, highly information-based and open system, diversity and forms of ecological aesthetics etc. With a multidimensional and composite concept, design view of the entire system complies with post-modern design trends and is involved with the overall consideration of design.

Maximum combination with the natural environment is pursued through design, and through the change of thinking modes and systematization ideas, the non-holistic and non-systematic design pattern can be changed by an ideological system that can better reflect cooperation, tolerance and sympathy. Pursuant to the system-theory principle of design, the whole process of the design can be externally associated with a "ring", and the design becomes annular. A systematic design shall be one that considers the whole process from the start of design to manufacture, distribution, utilization, waste disposition, decomposition, reuse until the product becomes a resource from the next cycle, i.e. the design principle of holistic system as required by ecological design is added. Therefore teaching of systematic design should be enhanced during design education to help students build the concept of holistic and systematic design from the very beginning.

\section{The Design View of Multiple Symbiosis}

"Symbiosis" refers to a state of cooperation, coexistence and mutual benefit. The whole world is multiple, rich and diversified. The "symbiosis" design view requires symbiosis between design and the natural environment and that the product should comply with the ecological law and be good for the healthy development of environment. The entire life cycle of the design system remains in a state of symbiosis from conception to raw materials and wastes.

The nature provides all-round services to humans, and through cooperation with the process and pattern to be followed by life, we can remarkably reduce the ecological impact of design, re-awaken the emotional tie between human the nature and create a symbiotic atmosphere among ecological environment, culture and design. In a natural system, the materials and energy flows in an end-to-end closed circulation composed of "source-consumption center-collection" where collection of the previous cycle serves as source of the follower cycle. The nature is in a state of multiple symbiosis. Therefore, new design concepts and theories such as to learn from nature, use nature as the background and seek common approaches to solve multiple different problems etc are what should be conveyed during design education at present.

\section{The Design View of Concerns on Ethics}

The concept of design ethics was proposed by Victor Papanek, a famous US design theorist who was of the opinion that the purpose of design is more than present functions, form and aim, and the more significant meaning of design lies in the fact that design embodies factors for formation of social systems. Therefore the consideration of design must cover short-term and long-term social factors. According to the ethics concept of ecological design, designers must consider the third world, people from developing countries, ecological balance, the utilization of limited resources on 
the earth and the protection of natural resources. Such consideration has greatly intensified the aspects of design thoughts and has promoted the development of design concept.

Papanek's view of limited resources has been generally acknowledged since the outbreak global energy crisis in the early 1970s. More and more designers, design theorists and educators are reevaluating design from the ethics angle, and ethics-concerned design is being brought into design education.

\section{The Design Concept Emphasizing Interaction and Participation}

Ecological design emphasizes that everyone is the designer and everyone participates in designing, and ecological design is a process of human-nature and human-human cooperation and interaction where the decision and choice made by each participant can profoundly affect the health of the entire environment and should be part of the ecological design. Therefore, ecological design is essentially contained in all daily behaviors of each one. This means, for professional designers, that the design must approach the public and the society so that knowledge of the mass can be dissolved into the design; at the same time, the designer's concept and objective of ecological design can be accepted by the public and become everyone's design and everyone's behavior. Therefore, the cultivation of interaction and participation is also important.

\section{Reform Measures for Design Education With Regard to Ecological Design}

The popularization and realization of ecological design concept cannot go without design education, and the concept and knowledge of ecological design should be brought systemically into teaching plans from the very beginning when art design courses are opened in universities and colleges, in order to assume the historical responsibility for the sustainable development of China.

\section{School and Teacher Oriented Reforms for Design Education}

\section{Specify the Position of Design}

The world can be likened to a net where each knot can be compared to a subject (or profession, industry, knowledge or individual), and when any knot is pulled, the rest knots can be affected. When a certain knot is used as the starting point for research (to start from a certain subject), the rest knots (subjects) then become the premise for connection of this knot; all knots (system elements) are contained in this net (system), and the making of each knot, therefore, cannot go without concerning other knots.

All subjects also form a net where all the subjects are correlated knots instead of separate knots. Knots are made to complete a net and make it functional, which shall apply to the net of the ecological system. Ecological design should follow the principle of systematic design, and as a knot in the ecological system, design plays a significant role. Being design imparters, teachers should first specify the position of design and try their best to build a research platform for ecological design from the angle of a specific subject; besides, teachers should enhance through design education the in-depth impact of ecological concepts on designers and specify the irreplaceable role played by design in ecological protection.

\section{Enhance Trans-Disciplinary Intersection and Fusion}

Involved with trans-disciplinary intersection and fusion, the settlement of ecological issues requires comprehensive control. To solve ecological issues concerned by design, we must approach or blend into such ecological issues from the angle of design on the basis of research achievements of other subjects, and any single subject can hardly solve ecological problems. Design is a subject of strong utility, and the fusion of design with other subjects needs more concern and enhancement. All objects are after all in widespread contact, and are interconnected and inter-constraint. We make classification to facilitate our research works, but our aim is to make clear the relation and comprehensively solve the problems, not merely classification researches. 


\section{Student Oriented Reforms for Design Education}

\section{Professional Literacy}

Designers should have a sympathetic heart to carefully feel each tiny process in life, and only when this sympathetic attitude is maintained in life and work can designers get familiar with the natural process and ecological process in specific designs and conscientiously join in the process of ecological design.

Works of a designer, on one hand, involve the development of products that comply with ecological requirements according to institutions and standards, and on the other hand, designers should constantly improve their ecological qualification and professional ethics and be active to further develop thrifty and educational consumer goods to guide the consumption and lead people to conscientiously increase the eco-awareness; hopefully a brand new life style in compliance with ecological requirements can be formed.

\section{Ability of Thinking}

Keen perceptibility can be acquired only through conscientious and active perception in daily lives. Works of art, living goods, natural articles, landscapes, humans and social phenomena all need earnestly comprehension. Ecological design requires strong abstracting ability in designers in order to grasp the essence of objects and such abstracting ability is the premise for summarizing and integrating abilities. System view aims to cultivate thinking habits to solve problems under restrictive conditions, and forms an important part of the new concept of ecological design. Creativity is to build a new order on the basis of negation and affirmation, the motive of human development and also a necessary condition for new ecological design.

\section{Build Correct Ecological Value}

Ecological value is a development value for respect on nature, care for ecology, protection of environment and harmony between human and nature. By carrying on the humanism value of modern culture, the ecological value affirms material and culture lives needed by humans and emphasizes the exertion of subjective initiative and creativity and that humans and the society are organic parts of the natural ecological system. The connotation and features of a scientific ecological value shall cover: a. Global species, ecological and environmental diversities are sacrosanct; b. Harmony between human and nature is a fundamental condition for human survival and development; c. the protection of ecology and environment is essentially in the realization of sustainable development. Design concepts and expression forms can be imperceptibly influenced only by building a correct ecological value.

In conclusion, ecological protection is a huge and formidable project, the realization of which has to rely on holistic endeavors of the entire society to build a 3D and all-round ecological system; and design shall play a very critical role. The design principle of the entire system, the design concept of regional, the design view with emphasis on ecological high-tech, emphasis on interaction and participation, emphasis on ethics, multiple symbiosis etc are all important. Ecological design is not a kind of luxury but a must, and it's a process, a kind of ethics, economic and must be esthetical. With its brand new design concept, ecological design has become an inexorable trend for the development of modern design. The function of design education in the popularization and realization of ecological design is beyond question, and only through active design education reforms, constant updating of design education concept, enhancement of trans-disciplinary intersection and fusion, cultivation of professional literacy and thinking ability in students to enable them build correct ecological value, emphasize regional cultures and learn the wise and rational application of new techniques can we gradually build the concept of ecological design and combine and advance the multiple factors including ecological concept, ecological techniques and ecological education etc. This is the only way we can develop and realize in an all-round way the formidable and high-minded mission of ecological design. 


\section{Bibliography}

[1] Ma Chundong, Ecological Design Published by Higher Education Press in August 2007

[2] Liu Xiaotao, Ecological Design Published by Shangdong Art Press in Jan. 2006

[3] Wang Shouzhi, A History of Modern Design Published by China Youth Press in 2002.

[4] Xu Ping and Pan Lin, Green Design Published by Jiangsu Art Press in 2001

[5] Alan Bauers [Britain], Nature in Design bytranslated by Wang Lifei and Liu Min and Wang Yan, Published by Jiangsu Art Press in 2001

[6] Zhou Xi and Li Zhandong, New Theory on Ecological Design--- Re-consideration about Ecological Design Pbulished by Southeast University Press in 2003 